\title{
Prevalence and distribution of endodontic treatments and apical periodontitis in an Italian population sample
}

European Journal of Inflammation 2016, Vol. I4(I) 48-53 (C) The Author(s) 2016 Reprints and permissions: sagepub.co.uk/journalsPermissions.nav DOI: $10.1177 / 1721727 \times 16638213$ eji.sagepub.com

\author{
M Dolci,' G Migliau, ${ }^{2}$ ZM Besharat, ${ }^{3}$ \\ LK Besharat ${ }^{2}$ and L Gallottini ${ }^{2}$
}

\begin{abstract}
This study was undertaken to investigate for the first time the distribution and prevalence of apical periodontitis and its determinants in an Italian population. A total of 312 participants ( 191 women, 121 men) received free clinical examination and full-mouth digital X-ray. Data regarding the oral health status of the patients were collected such as the reason for the visit and the incidence of lesions. We examined $810 \mathrm{l}$ teeth, $6.59 \%$ of which had been submitted to endodontic treatment and $15.02 \%$ of which were lower molars. Lower molars were reported missing more frequently, in detail $16.24 \%$. Moreover $3.89 \%$ of the lower molars had developed an apical lesion, whereas only $0.08 \%$ of the lower incisors. Periapical lesions were present in $0.29 \%$ of not endodontically treated teeth, while in endodontically treated teeth lesions were present in 17.04\%. Additionally, 15.65\% of the endodontically treated teeth showed incongruous (long or short) seals. A prevalence of women (6I.21\%) over men (38.78\%) was reported indicating that women attend to their oral health more. Endodontic treatment success and failure in over- or under-filled teeth was evaluated with success being verified in $97 \%$ of appropriately treated teeth; the success rate decreases in inappropriately treated teeth (by $72.73 \%$ in over-filled and $77.71 \%$ under-filled teeth). The prevalence of teeth with periapical lesions amounted to $1.35 \%$. Finally, endodontically treated teeth with periapical lesions amounted to $17.04 \%$. Successful treatment can be achieved when disease is intercepted at an early stage while prevention still remains better than cure.
\end{abstract}

\section{Keywords}

apical periodontitis, Italian population sample, oral health

Date received: I5 March 2015; accepted: I2 February 2016

\section{Introduction}

Apical periodontitis (AP) represents a periapical inflammation in response to long-standing, mostly microbial irritation from the root canal. Clinical diagnosis of AP requires assessment of the patient's symptoms, signs, and radiographs.

The radiographic appearance of AP is usually a radiolucent area of variable size at the apex of the affected root, although occasionally, radioopaque zones can be seen. Interpretation of radiographs, either periapical, panoramic, or a combination of panoramic and periapical, is the only method that can be used in an epidemiological study when evaluating AP.
The purpose of this study is to gain knowledge of distribution and prevalence of AP and its determinants including treatment outcome in an Italian

\footnotetext{
'Department of Oral Sciences, Dental School, University G.

D’Annunzio, Chieti, Italy

2Department of Oral and Maxillo-facial Sciences, "Sapienza" University of Rome, Italy

${ }^{3}$ Department of Molecular Medicine, "Sapienza” University of Rome, Italy

Corresponding author:

LK Besharat, Department of Oral and Maxillo-facial Sciences, Sapienza University of Rome, Viale Regina Elena, 287/A, 0016I Rome, Italy. Email: besharatlk84@yahoo.it
} 
population sample evaluated by presence or absence of AP using a retrospective analysis of orthopantomograms (OPGs).

The assessment of the incidence and prevalence of chronic AP may help to define treatment needs and to relate treatment outcome to various technical and clinical factors of endodontic intervention.

Since this is the first study of this kind to be conducted in Italy, the results obtained were compared with those produced by similar studies published in the international literature.

\section{Materials and methods}

\section{Selection of the sample}

The sample consisted of patients who received free clinical examination and full-mouth digital $\mathrm{X}$-ray at a private clinic in Rome between 24 September 2007 and 5 March 2008. In all cases, in addition to the examination and X-ray, personal data were collected.

The fact that the screening was completely free of charge guaranteed a heterogeneous sample in terms of socioeconomic status and oral health. Indeed, the examinations, not necessarily conducted in the presence of pain, involved both dentally healthy participants and patients affected by different dental diseases.

Of the 385 individuals, 73 were excluded from the study analysis because they were still in the primary or mixed dentition stage, or because they had fewer than nine teeth. The participants with fewer than nine teeth were excluded because in such cases there is a very strong likelihood of periodontal impairment, which could lead to periapical lesions of periodontal origin mistakenly being considered lesions of endodontic origin, and vice versa. Third molars were not considered in this study, on account of their high anatomical and morphological variability.

The final study sample included 312 participants, 191 girls/women (mean age, 44.24 years) and 121 boys/men (mean age, 41.49 years), with an age range of 11-79 years.

\section{$X$-rays and assessment of periapical status}

The digital X-rays analyzed in this study were taken using a Gendex Orthoralix 9200 DDE (Dental System, Des Plaines, IL, USA), connected to a personal computer installed with the
VixWin (trademark of the Gendex Corporation) application software.

The X-rays were all taken by the same operator; in order to achieve the same patient set-up, guaranteeing homogeneous results.

The full-mouth X-ray is often used in epidemiological studies. The fact that it allows all the teeth to be visualized at the same time on a single radiograph, reducing the radiation exposure of the patient and allowing faster execution of the procedure, makes it more advantageous than a fullmouth of intra-oral X-rays, including bitewing and periapical X-rays.

In this study, all the images were found to be of excellent quality. Of the 8101 teeth studied, only 10 (4 upper incisors, 2 upper canines, and 4 upper premolars) were excluded from the statistical calculations due to lack of image clarity.

Periapical status was assessed using a periapical index (PAI) scoring system ${ }^{1}$ which was used in other similar investigations. ${ }^{2}$ The PAI system provides criteria that are measurable (scores are related to reference radiographs), mutually exclusive (when in doubt, the higher value is assigned), meaningful (based on radiographic evaluation of the periradicular tissues), and reproducible (Krstavik et al.1986).

Each root was classified as:

1. Normal periapical structures;

2. Small changes in bone structure;

3. Changes in bone structure with some mineral loss;

4. Periodontitis with well-defined radiolucent area;

5. Severe periodontitis with exacerbating features.

Each PAI category represents a step on a sequential scale of periapical infection. In multiradicular teeth, the score assigned is that of the root with the highest PAI. ${ }^{1}$

The reliability of the results was ascertained by using two observers and computing Cohen's kappa for reliability assessment. The mean reliability was found to be 0.813 .

\section{Data collection}

The 191 women (age range, 15-79 years) were divided into the following age groups: 15-29 years 
(28 patients); 30-39 years (53 patients); 40-49 years (41 patients); 50-59 years (15 patients); and over 60 years (41 patients). Due to incompleteness of personal data provided at the initial examination, the ages of 13 women were not reported. These participants, together with one of the men, were categorized as "age unknown".

The 121 men were also divided into age groups: 15-29 years (23 patients); 30-39 years (37 patients); $40-49$ years (33 patients); $50-59$ years ( 8 patients); and over 60 years (19 patients).

To obtain a complete picture of the oral health status of this population and to investigate, in detail, the relationship between periapical lesions and quality of previous endodontic treatments, a table of data on root filling lengths was created, considering all filled teeth. These data were then compared with data on the incidence of lesions in teeth previously submitted to endodontic treatment.

We also evaluated the incidence of periapical lesions in untreated teeth, which, very interestingly, was found to be lower than the incidence of these lesions in endodontically treated teeth.

Furthermore, analysis of the reasons for the consultation, noted on each patient's acceptance chart at the initial examination, highlighted the presence of a relationship between periapical or radiographically appreciable carious lesions and the reporting of pain or discomfort as the main reason for the consultation. However, it was also interesting to examine the other reasons that brought these patients to our attention. On the basis of the information collected, we identified six main reasons for these patients' attendance to our clinic:

1. Tartar removal or periodontal problems (7.69\%);

2. Specialist orthodontic examinations $(8.01 \%)$;

3. Presence of pain $(1.92 \%)$;

4. Prosthetic, or implant-prosthetic reasons $(1.92 \%)$

5. Treatments (fillings, reconstructions) (3.2\%);

6. Checkups (77.24\%).

\section{Results}

The analytical approach adopted in this study took the form of observation and critical analysis of a set of tables created on the basis of direct observations of the X-rays.
The study sample comprised 312 individuals, 191 women ( $61.21 \%$ of the total) and 121 men (38.78\%). Dividing the patients by age, it was found that the individuals aged 30-39 years, both men and women, were the largest group of participants attending the clinic (90 individuals, 28.84\% of the total); instead, the smallest group, again for both sexes, was that of the individuals aged 50-59 years (24 patients, $7.69 \%$ of the total).

The general values gathered from an initial investigation with the aim being to provide an overview of the population's oral health status taking different aspects into account will be outlined now. From this initial analysis it emerged that the teeth most often missing were the lower molars (16.24\%). Another interesting finding concerned the prevalence of intact teeth, i.e. teeth that have not been submitted to prosthetic, conservative, or endodontic treatments and that show no carious or periodontal/apical lesions; this prevalence was $94.72 \%$ in the lower incisors, a finding born out of the data on the prevalence of caries and fillings in untreated teeth, which, in both cases, was highest in the molars with $8.36 \%$ and $35.74 \%$, respectively.

Of the total 8101 teeth examined, $6.59 \%$ had been submitted to endodontic treatment, $15.02 \%$ of which were lower molars. In the endodontically treated teeth, the prevalence of teeth presenting an apical lesion was found to range from a maximum of $3.89 \%$ in the lower molars to a minimum of $0.08 \%$ in the lower incisors.

The absolute values of the various characteristics examined previously were determined. The total number of teeth was 8101 , making it easier to extract the percentages, in the entire population, of missing teeth $(7.91 \%)$, carious teeth $(3.45 \%)$; filled teeth $(13.71 \%)$, crowned teeth $(4.18 \%)$, and teeth with periapical lesions $(0.27 \%)$; in the endodontically treated teeth, we calculated the numbers and thus the percentages of teeth presenting osteolytic periapical lesions $(1.12 \%)$, teeth with amalgam or composite fillings $(1.74 \%)$, and crowned teeth $(4.93 \%)$ (Table 1$)$.

Periapical status in the population is detailed in Table 2. AP was present in 113 of the 8101 teeth examined (1.39\%). Of the 7567 teeth that had not been submitted to endodontic treatment, 22 $(0.29 \%)$ showed periapical lesions. Of the 534 endodontically treated teeth, 91 (17.04\%) showed lesions. 
Table I. Characteristics of studied population.

\begin{tabular}{|c|c|c|c|c|c|c|c|c|}
\hline & & \multicolumn{7}{|c|}{ Endodontically treated } \\
\hline & & \multirow[b]{2}{*}{$\mathrm{n}$} & \multicolumn{2}{|c|}{ Lesioned } & \multicolumn{2}{|c|}{ Filled } & \multicolumn{2}{|c|}{ Crown } \\
\hline & & & $\mathrm{n}$ & $\%$ & $n$ & $\%$ & $\mathrm{n}$ & $\%$ \\
\hline \multirow{4}{*}{ Upper } & Inc. & 47 & 4 & $8.51 \%$ & 14 & $29.78 \%$ & 33 & $70.21 \%$ \\
\hline & Can. & 40 & 2 & $5.00 \%$ & 5 & $12.50 \%$ & 36 & $90.00 \%$ \\
\hline & Prem. & 100 & 14 & $14.00 \%$ & 18 & $1.80 \%$ & 80 & $80.00 \%$ \\
\hline & Molars & 115 & 13 & $11.30 \%$ & 33 & $28.69 \%$ & 88 & $76.52 \%$ \\
\hline \multirow[t]{4}{*}{ Lower } & Inc. & 9 & 1 & II.II\% & 4 & $44.44 \%$ & 3 & $33.33 \%$ \\
\hline & Can. & 11 & 2 & $18.18 \%$ & 0 & $0.00 \%$ & 10 & $90.90 \%$ \\
\hline & Prem. & 65 & 14 & $21.53 \%$ & 16 & $24.61 \%$ & 48 & $73.84 \%$ \\
\hline & Molars & 147 & 41 & $27.89 \%$ & 51 & $34.69 \%$ & 80 & $54.42 \%$ \\
\hline
\end{tabular}

Inc: Incisor, Can: Canine, Prem: Premolar.

Table 2. Periapical status of population.

\begin{tabular}{llll}
\hline & Teeth with lesions & & $\mathrm{n}$ \\
\cline { 2 - 4 } & TOT & 113 & $\%$ \\
\hline Examined teeth & 8101 & 22 & $1.39 \%$ \\
Teeth not endodontically treated & 7567 & 91 & $0.29 \%$ \\
Endodontically treated teeth & 534 & $17.04 \%$ \\
\hline
\end{tabular}

In the endodontically treated teeth, periapical lesions were most likely to affect lower molars $(27.89 \%)$, in which they were considerably more frequent than in other teeth.

Table 3, like Table 2, investigates periapical status, but this time subdividing the sample by sex and age. It emerges that the highest percentage of periapical lesions is found in women aged 50-59 years $(2.82 \%)$, whereas in the men, the over- 60 s are the age group most affected $(2.56 \%)$.

Table 4 provides a picture of the quality of the endodontic treatments carried out in the sample. From this analysis it was found that of 534 endodontically treated teeth, $74(13.60 \%)$ had a short filling in relation to the apex, of which $45.94 \%$ were lower molars; and $11(2.02 \%)$ had a long root canal filling, of which $27.27 \%$ were upper canines. In total, $85(15.65 \%)$ of the endodontically treated teeth showed incongruous (long or short) seals.

We also considered data on the prevalence of participants with complete, intact dentition, i.e. the percentages of men and women who had never undergone any dental procedure, prosthetic, conservative, or endodontic and who still had a full set of teeth (with or without the third molar). Of the 312 participants examined, $49(15.70 \%)$ showed these characteristics, $31(16.23 \%)$ of the 191 women and $18(14.87 \%)$ of the 121 men.

\section{Discussion and conclusions}

Analyzing the sample, we found a prevalence of women $(61.21 \%)$ over men $(38.78 \%)$. This finding, also present in other studies, could reflect a greater level of interest in oral health among women. However, the present study, like other similar ones, shows that there is no substantial gender difference in the prevalence of endodontic treatments $(6.66 \%$ in women, $6.54 \%$ in men) or of endodontic lesions (1.59\% in women and $1.08 \%$ in men).

Analysis of Table 5, which sets out the results of numerous studies on the prevalence of periapical lesions and endodontic treatments published in the international literature, reveals that the data produced by our study are not entirely superimposable on those of previous studies. All the literature studies showed a significantly increased frequency of periapical lesions in endodontically treated teeth. This aspect, central to our research study, was one of the few that allowed us to compare our study with all the studies present in the literature. In our study, unlike the others, we tried to highlight a number of aspects that have often not been taken into consideration in the international literature.

The percentages of endodontic treatment success and failure in over- or under-filled teeth that we found in this study are perfectly in line with 
Table 3. Periapical status of population by sex and age.

\begin{tabular}{|c|c|c|c|c|c|c|c|c|c|c|}
\hline & \multirow[t]{3}{*}{ Age range (years) } & \multicolumn{3}{|c|}{ Examined teeth } & \multicolumn{3}{|c|}{ Untreated teeth } & \multicolumn{3}{|c|}{ Treated teeth } \\
\hline & & \multirow[t]{2}{*}{ Total } & \multicolumn{2}{|c|}{ Lesioned } & \multirow[t]{2}{*}{ Total } & \multicolumn{2}{|c|}{ Lesioned } & \multirow[t]{2}{*}{ Total } & \multicolumn{2}{|c|}{ Lesioned } \\
\hline & & & $\mathrm{n}$ & $\%$ & & $\mathrm{n}$ & $\%$ & & $\mathrm{n}$ & $\%$ \\
\hline \multirow[t]{6}{*}{ Women } & $15-29$ & 778 & 7 & $0.89 \%$ & 760 & I & $0.13 \%$ & 14 & 6 & $42.85 \%$ \\
\hline & $30-39$ & 1439 & 17 & $1.18 \%$ & $137 \mid$ & 1 & $0.07 \%$ & 64 & 16 & $25 \%$ \\
\hline & $40-49$ & 1124 & 12 & $1.06 \%$ & 1051 & 0 & $0 \%$ & 69 & 12 & $17.39 \%$ \\
\hline & $50-59$ & 390 & II & $2.82 \%$ & 329 & 2 & $0.60 \%$ & 57 & 9 & $15.78 \%$ \\
\hline & $>60$ & 972 & 24 & $2.46 \%$ & 864 & 4 & $0.46 \%$ & 103 & 20 & $19.41 \%$ \\
\hline & Age unknown & 250 & 8 & $3.20 \%$ & 217 & 1 & $0.46 \%$ & 26 & 7 & $26.92 \%$ \\
\hline \multirow[t]{9}{*}{ Men } & $15-29$ & 629 & 3 & $0.47 \%$ & 613 & 0 & $0 \%$ & 16 & 3 & $18.75 \%$ \\
\hline & $30-39$ & 973 & 5 & $0.51 \%$ & 934 & 2 & $0.21 \%$ & 39 & 3 & $7.69 \%$ \\
\hline & $40-49$ & 901 & 12 & $1.33 \%$ & 832 & 3 & $0.36 \%$ & 66 & 9 & $13.63 \%$ \\
\hline & $50-59$ & 191 & 3 & $1.57 \%$ & 153 & 2 & $1.30 \%$ & 38 & I & $2.63 \%$ \\
\hline & $>60$ & 429 & II & $2.56 \%$ & 389 & 6 & $1.54 \%$ & 43 & 5 & $11.62 \%$ \\
\hline & Age unknown & 25 & 0 & $0 \%$ & 25 & 0 & $0 \%$ & 0 & 0 & $0 \%$ \\
\hline & Women & 4953 & 79 & & 4592 & 9 & & 333 & 70 & \\
\hline & Men & 3148 & 34 & & 2946 & 13 & & 202 & 21 & \\
\hline & Total & 8101 & 113 & & 7538 & 22 & & 535 & 91 & \\
\hline
\end{tabular}

Table 4. Quality of endodontic treatments.

\begin{tabular}{|c|c|c|c|c|c|c|c|c|c|c|}
\hline \multirow[b]{3}{*}{ Upper } & \multicolumn{10}{|c|}{ Distance from apex } \\
\hline & \multirow[b]{2}{*}{ Inc. } & \multirow{2}{*}{$\frac{\text { Total }}{47}$} & \multicolumn{2}{|c|}{ Short } & \multicolumn{2}{|c|}{ Long } & \multicolumn{2}{|c|}{ Correct } & \multicolumn{2}{|c|}{ Incongruous } \\
\hline & & & 2 & $4.25 \%$ & 2 & $4.25 \%$ & 43 & $91.48 \%$ & 4 & $8.51 \%$ \\
\hline & Can. & 50 & 1 & $2.00 \%$ & 3 & $6.00 \%$ & 36 & $72.00 \%$ & 4 & $8.00 \%$ \\
\hline & Prem. & 100 & II & $11.00 \%$ & I & $1.00 \%$ & 88 & $88.00 \%$ & 12 & $12.00 \%$ \\
\hline & Molars & 115 & 11 & $9.56 \%$ & 2 & $1.73 \%$ & 102 & $88.69 \%$ & 13 & $11.30 \%$ \\
\hline \multirow[t]{5}{*}{ Lower } & Inc. & 9 & 0 & $0.00 \%$ & 0 & $0.00 \%$ & 9 & $100.00 \%$ & 0 & $0.00 \%$ \\
\hline & Can. & II & 0 & $0.00 \%$ & 0 & $0.00 \%$ & 11 & $100.00 \%$ & 0 & $0.00 \%$ \\
\hline & Prem. & 65 & 15 & $23.07 \%$ & 2 & $3.07 \%$ & 48 & $73.84 \%$ & 17 & $26.14 \%$ \\
\hline & Molars & 147 & 34 & $23.12 \%$ & I & $0.68 \%$ & 112 & $76.19 \%$ & 35 & $23.80 \%$ \\
\hline & & 544 & 74 & $13.60 \%$ & 11 & $2.02 \%$ & 449 & $82.53 \%$ & 85 & $15.65 \%$ \\
\hline
\end{tabular}

Inc: Incisor, Can: Canine, Prem: Premolar.

what is reported in the scientific literature, with success being verified in $97 \%$ of appropriately treated teeth; the success rate falls in inappropriately treated teeth (by $72.73 \%$ in over-filled and $77.71 \%$ in under-filled teeth).

Still with reference to Table 5, it can be observed that the prevalence of teeth with periapical lesions found in the present study $(1.35 \%$, corresponding to 110 of the total 8101 teeth) falls within the values reported in the literature, which range from $0.60 \%$, in the Norwegian study of $1995,{ }^{3}$ to $9.8 \%$, in the 1968 Swedish study. ${ }^{4}$

The situation is different in regards to the prevalence of periapical lesions in endodontically treated teeth. Of the 534 endodontically treated teeth present in our sample, 91 had lesions, which corresponds to $17.04 \%$, a value very different from those reported in the literature, which range from $61 \%$ in the German study of $1997^{13}$ to $25 \%$ in the Irish study of 2000.5

In conclusion, one consideration prompted by this study, which is worth underlining, is the importance of prevention. Indeed, the most stimulating aspect of this research was the awareness that we were investigating a sample of participants who were being examined by us free of charge; since we were thus able to observe a population sample in its actual oral and dental state, this investigation assumed the value of a screening study.

It is to be hoped that this kind of approach might also be applied within public healthcare organizations / hospitals, which are an important resource 
Table 5. Results comparison with international studies.

\begin{tabular}{|c|c|c|c|c|}
\hline \multirow[t]{2}{*}{ Study (year) } & \multirow[t]{2}{*}{ Country } & \multirow[t]{2}{*}{ Teeth $(n)$} & \multirow{2}{*}{$\frac{\text { Teeth with lesions }}{\%}$} & \multirow{2}{*}{$\frac{\text { Endo. treated teeth }}{\text { Teeth with lesions (\%) }}$} \\
\hline & & & & \\
\hline Petterson et al. $(1986)^{6}$ & Sweden & 4985 & $6.60 \%$ & $31.00 \%$ \\
\hline Allard and Palmqvist $(1968)^{4}$ & Sweden & 2567 & $9.80 \%$ & $27.00 \%$ \\
\hline Eckerbom et al. $(1987)^{7}$ & Sweden & 4889 & $5.20 \%$ & $26.00 \%$ \\
\hline Odesjo et al. $(1990)^{8}$ & Sweden & 17430 & $2.90 \%$ & $25.00 \%$ \\
\hline Eriksen and Bjertness $(1991)^{9}$ & Norway & 2940 & $3.50 \%$ & $36.60 \%$ \\
\hline DeCleen et al. $(1993)^{10}$ & Holland & 4196 & $4.50 \%$ & $39.00 \%$ \\
\hline Eriksen et al. $(1995)^{3}$ & Norway & 3282 & $0.60 \%$ & $38.00 \%$ \\
\hline Buckley and Spangberg (1995) ${ }^{\prime \prime}$ & USA & 5272 & $4.10 \%$ & $31.30 \%$ \\
\hline Weiger et al. (1997) 12 & Germany & 7897 & $3.00 \%$ & $61.00 \%$ \\
\hline Sidarivacius et al. (1999)13 & Lithuania & 3892 & $7.20 \%$ & $35.00 \%$ \\
\hline Loftus et al. $(2000)^{5}$ & Ireland & 7424 & $2.00 \%$ & $25.00 \%$ \\
\hline Present study (2008) & Italy & 8101 & $1.35 \%$ & $17.04 \%$ \\
\hline
\end{tabular}

for the entire population. This could also lead to reductions in public spending, in accordance with the old adage "prevention is better than cure." Early interception of disease states is the only means of increasing the chances of treatment success; furthermore, in many cases this would drastically reduce the cost for the patient, an aspect that, in the public health sector, would translate into considerably shorter waiting lists and lower costs.

\section{Declaration of conflicting interests}

The authors declared no potential conflicts of interest with respect to the research, authorship, and/or publication of this article.

\section{Funding}

This research received no specific grant from any funding agency in the public, commercial, or not-for-profit sectors.

\section{References}

1. Ørstavik D, Kerekes K and Eriksen HM (1986) The periapical index: A scoring system for radiographic assessment of apical periodontitis. Endodontics \& Dental Traumatology 2: 20-34.

2. Dugas NN, Lawrence HP, Teplitsky PE et al. (2003) Periapical health and treatment quality assessment of root-filled teeth in two Canadian populations. International Endodontic Journal 36: 181-192.

3. EriksenHM, BersetGP, Hansen BF etal.(1995)Changes in endodontic status 1973-1993 among 35-year-olds in Oslo, Norway. International Endodontic Journal 28: 129-132.

4. Allard U and Palmqvist S (1986) A radiographic survey of periapical conditions in elderly people in a Swedish county population. Endodontics \& Dental Traumatology 2: 103-108.
5. Loftus JJ, Keating AP and McCartan BE (2005) Periapical status, prevalence and quality of endodontic treatment in an adult Irish population. International Endodontic Journal 38: 81-86.

6. Petterson K, Petterson A, OlssonBetal.(1986)Technical quality of root fillings in an adult Swedish population. Endodontic \& Dental Traumatology 2: 99-102.

7. Eckerbom M, Andersson JE and Magnusson T (1989) A longitudinal study of changes in frequency and technical standard of endodontic treatment in Swedish population. Endodontics \& Dental Traumatology 5: 27-31.

8. Odesjo B, Hellden L, Salonen L et al. (1990) Prevalence of previous endodontic treatment, technical standard and occurrence of periapical lesions in arandomly selected adult, general population. Endodontics \& Dental Traumatology 6: 265-272.

9. Eriksen HM and Bjertness E (1991) Prevalence of apical periodontitis and results of endodontic treatment in middle-age adults in Norway. Endodontics \& Dental Traumatology 7: 1-4.

10. De Cleen MJ, Schuurs AH, Wesselink PR et al. (1993) Periapical status and prevalence of endodontic treatment in an adult Dutch population. International Endodontic Journal 26: 112-119.

11. Bukley M and Spanberg LS (1995) The prevalence and technical quality of endodontic treatment in an American subpopulation. Oral Surgery, Oral Medicine, Oral Pathology Oral Radiology, and Endodontics 79: 92-100.

12. Weiger R, Hitzler S, Hermle G et al. (1997) Periapical status, quality of root canal fillings and estimated endodontics treatment needs in an urban German population. Endodontics \& Dental Traumatology 13: 69-74.

13. Sidaravicius B, Aleksejuniene J and Eriksen HM (1999) Endodontic treatment and prevalence of apical periodontitis in an adult population of Vilnius, Lithuania. Endodontics \& Dental Traumatology 15: 210-215. 